\title{
On Memory-Bound Functions for Fighting Spam
}

\author{
Cynthia Dwork ${ }^{1}$, Andrew Goldberg ${ }^{1}$, and Moni Naor ${ }^{2 \star}$ \\ 1 Microsoft Research, SVC \\ 1065 L'Avenida \\ Mountain View, CA 94043 \\ \{dwork, goldberg\}@microsoft.com \\ 2 Weizmann Institute of Science \\ Rehovot 76100, Israel \\ naor@wisdom. weizmann.ac.il
}

\begin{abstract}
In 1992, Dwork and Naor proposed that e-mail messages be accompanied by easy-to-check proofs of computational effort in order to discourage junk e-mail, now known as spam. They proposed specific CPU-bound functions for this purpose. Burrows suggested that, since memory access speeds vary across machines much less than do CPU speeds, memory-bound functions may behave more equitably than CPU-bound functions; this approach was first explored by Abadi, Burrows, Manasse, and Wobber [3].

We further investigate this intriguing proposal. Specifically, we

1. Provide a formal model of computation and a statement of the problem;

2. Provide an abstract function and prove an asymptotically tight amortized lower bound on the number of memory accesses required to compute an acceptable proof of effort; specifically, we prove that, on average, the sender of a message must perform many unrelated accesses to memory, while the receiver, in order to verify the work, has to perform significantly fewer accesses;

3. Propose a concrete instantiation of our abstract function, inspired by the RC4 stream cipher;

4. Describe techniques to permit the receiver to verify the computation with no memory accesses;

5. Give experimental results showing that our concrete memory-bound function is only about four times slower on a $233 \mathrm{MHz}$ settop box than on a $3.06 \mathrm{GHz}$ workstation, and that speedup of the function is limited even if an adversary knows the access sequence and uses optimal off-line cache replacement.
\end{abstract}

\section{Introduction}

Unsolicited commercial e-mail, or spam, is more than just an annoyance. At two to three billion daily spams worldwide, or close to $50 \%$ of all e-mail, spam incurs huge infrastructure costs, interferes with worker productivity, devalues the internet, and is ruining e-mail.

\footnotetext{
* Incumbent of the Judith Kleeman Professorial Chair. Research supported in part by a grant from the Israel Science Foundation. Part of this work was done while visiting Microsoft Research, SVC.
} 
This paper focuses on the computational approach to fighting spam, and, more generally, to combating denial of service attacks, initiated by Dwork and Naor [11] (also discussed by Back; see [18,9]). The basic idea is:

"If I don't know you and you want to send me a message, then you must prove that you spent, say, ten seconds of CPU time, just for me and just for this message."

The "proof of effort" is cryptographic in flavor; as explained below, it is a moderately hard to compute (but very easy to check) function of the message, the recipient's address, and a few other parameters. Dwork and Naor called such a function a pricing function because the proposal is fundamentally an economic one: machines that currently send hundreds of thousands of spam messages each day, could, at the 10-second price, send only eight thousand. To maintain the current 2-3 billion daily messages, the spammers would require 250,000-375,000 machines.

CPU-bound pricing functions suffer from a possible mismatch in processing speeds among different types of machines (desktops $v s$. servers), and in particular between old machines and the presumed new, top of the line, machines that could be used by a high-tech spam service. In order to remedy these disparities, Burrows proposed an alternative computational approach, first explored in [3], based on memory latency. His creative suggestion is to design a pricing function requiring a moderately large number of scattered memory accesses. Since memory latencies vary much less across machines than do clock speeds, memory-bound functions should prove more equitable than CPUbound functions.

Our Contributions. In the current paper we explore Burrows' suggestion. After reviewing the computational approach (Section 2) and formalizing the problem (Section 3), we note that the known time/space tradeoffs for inverting one-way functions [21,14] (where space now refers to cache) constrain the functions proposed in [3] (Section 4). We propose an abstract function, using random oracles, and give a lower bound on the amortized complexity of computing an acceptable proof of effort (Section 51]. We suggest a very efficient concrete implementation of the abstract function, inspired by the RC4 stream cipher (Section 6). We present experimental results showing that our concrete memorybound function is only about four times slower on a $233 \mathrm{MHz}$ settop box than on $3.06 \mathrm{GHz}$ workstation (Section [7). Finally, we modify our concrete proposal to free the receiver from having to make memory accesses, with the goal of allowing small-memory devices to be protected by our computational anti-spam protocol. A more complete version of the paper is available at

www.wisdom. weizmann.ac.il/ naor/PAPERS/dgn.html.

\section{Review of the Computational Approach}

In order to send a message $m$, software operating on behalf of the sender computes a proof of computational effort $z=f(m$, sender, receiver, date) for a moderately hard

\footnotetext{
${ }^{1}$ None of [11,18993] obtains a lower bound.
} 
to compute "pricing" function $f$. The message $m$ is transmitted together with the other arguments to $f$ and the resulting proof of effort 2 . Software operating on behalf of the receiver checks that the proof of effort has been properly computed; a missing proof can result in some user-prespecified action, such as placing the message in a special folder, marking it as spam, subjecting it to further filtering, and so on. Proof computation and verification should be performed automatically and in the background, so that the typical user e-mail experience is unchanged.

The function $f$ is chosen so that (1) $f$ is not amenable to amortization; in particular, computing $f(m$, sender, Alice, date $)$ does not help in computing $f(m$, sender, Bob, date). This is key to fighting spam: the function must be recomputed for each recipient (and for any other change of parameters). (2) There is a "hardness" parameter to vary the cost of computing $f$, allowing it to grow as necessary to accommodate Moore's Law. (3) There is an important difference in the costs of computing $f$ and of checking $f$ : the cost of sending a message should grow much more quickly as a function of the hardness parameter than the cost of checking that a proof of effort is correct. This allows us to keep verification very cheap, ensuring that the ability to wage a denial of service attack against a receiver is not exacerbated by the spam-fighting tool. In addition, if verification is sufficiently cheap, then it can be carried out by the receiver's mail (SMTP) server.

Remark 1. With the right architecture, the computational approach permits single-pass send-and-forget e-mail: once the mail is sent the sender never need take any further action, once the mail is received the proof of effort can be checked locally; neither sender nor receiver ever need contact a third party. In other words, single-pass send-and-forget means that e-mail, the killer application of the Internet, is minimally disturbed.

Remark 2. We briefly remark on our use of the date as an argument to the pricing function. The receiver temporarily stores valid proofs of effort. The date is used to control the amount of storage needed. When a new proof of effort, together with its parameters, is received, one first checks the date: if the date is, say, over a week old, then the proof is rejected. Otherwise, the receiver checks the saved proofs of effort to see if the newly received proof is among them. If so, then the receiver rejects the message as a duplicate. Otherwise, the proof is checked for validity.

In [11], $f$ is a forged signature in a careful weakening of the Fiat-Shamir signature scheme. Back's proposal, called HashCash, is based on finding hash collisions. It is currently used to control access to bulletin boards [18]; verification is particularly simple in this scheme.

\section{Computational Model and Statement of the Problem}

The focus on memory-bound functions requires specification of certain details of a computational model not common in the theory literature. For example, in real contemporary

\footnotetext{
${ }^{2}$ Having $m$ as an argument to the function introduces some practical difficulties in real mail systems. One can instead use the following three arguments: receiver's e-mail address, date, and a nonce. However, the intuition is more clear if we include the message.
} 
hardware there is (at least) two kinds of space: ordinary memory (the vast majority) and cache - a small amount of storage on the same integrated circuit chip as the central processing unit3. Cache can be accessed roughly 100 times more quickly than ordinary memory, so the computational model needs to differentiate accordingly. In addition, when a desired value is not in cache (a cache miss), and an access to memory is made, a small block of adjacent words (a cache line), is brought into the cache simultaneously. So in some sense values nearby the desired one are brought into cache "for free". Our model is an abstraction that reflects these considerations, among others.

When arguing the security of a cryptographic scheme one must specify two things: the power of the adversary and what it means for the adversary to have succeeded in breaking the scheme. In our case defining the adversary's power is tricky, since we have to consider many possible architectures. Nevertheless, for concreteness we assume the adversary is limited to a "standard architecture" as follows:

1. There is a large memory, partitioned into $m$ blocks (also called cache lines) of $b$ bits each;

2. The adversary's cache is small compared to the memory. The cache contains at most $s$ (for "space") words; a cache line typically contains a small number (for example, 16) of words;

3. Although the memory is large compared to the cache, we assume that $m$ is still only polynomial in the largest feasible cache size $s$;

4. Each word contains $w$ bits (commonly, $w=32$ );

5. To access a location in the memory, if a copy is not already in the cache (a cache miss), the contents of the block containing that location must be brought into the cache - a fetch; since every cache miss results in a fetch, we use these terms interchangeably;

6. We charge one unit for each fetch of a memory block. Thus, if two adjacent blocks are brought into cache, we charge two units (there is no discount for proximity at the block level).

7. Computation on data in the cache is essentially free. By not (significantly) charging the adversary for this computation, we are increasing the power of the adversary; this strengthens the lower bound.

Thus, the challenge is to design a pricing function $f$ as described in Section 2 together with algorithms for computing and checking $f$, in which the costs of the algorithms are measured in terms of memory fetches and the "real" time to compute $f$ on currently available hardware is, say, about 10 seconds (in fact, $f$ may be parameterized, and the parameters tuned to obtain a wide range of target computation times).

The adversary's goal is to maximize its production of (message, proof of computational effort) pairs while minimizing the number of cache misses incurred. The adversary is considered to have won if it has a strategy that produces many (message, proof) pairs with an amortized number of fetches (per message plus proof) which is substantially less than the expected number of fetches for a single computation obtained in the analysis of the algorithm. We do not care if the messages are sensical or not.

We remark that it may be possible to defeat a memory-bound function with specific parameters by building a special-purpose architecture, such as a processor with a huge,

\footnotetext{
${ }^{3}$ In fact, there are multiple levels of cache; Level 1 is on the chip.
} 
fast, on-chip cache. However, since the computational approach to fighting spam is essentially an economic one, it is important to consider the cost of designing and building the new architecture. These issues are beyond the scope of this paper.

\section{Simple Suggestions and Small-Space Cryptanalyses}

In the full paper we show that pricing functions based on meet in the middle and subset sum can be computed with very few memory access, and hence do not solve our problem. In these proceedings we confine our attention to the proposal in [3], described next.

Easy-to-Compute Functions. These functions are essentially iterates of a single basic "random-looking" function $g$. They vary in their choice of basic function. The basic function has the property that a single function inversion is more expensive than a memory look-up.

Let $n$ and $\ell$ be parameters and let $g:\{0,1\}^{n} \longrightarrow\{0,1\}^{n}$. Let $g_{0}$ be the identity function and for $i=1 \ldots \ell$, let the function $g_{i}(x)=g\left(g_{i-1}(x)\right) \oplus i$. Input. $y=g_{\ell}(x)$ for some $x \in\{0,1\}^{n}$ and $\alpha$, a hash of the values $x, g_{1}(x), \ldots, g_{\ell}(x)$. Output. $x^{\prime} \in g_{\ell}^{-1}(y)$ such that the string $x^{\prime}, g_{1}\left(x^{\prime}\right), \ldots, g_{\ell}\left(x^{\prime}\right)$ hashes to $\alpha$.

The hope is that the best way to resolve the challenge is to build a table for $g^{-1}$ and to work backwards from $y$, exploring the tree of pre-image 4 . Since forward computation of $g$ is assumed to be quite easy, constructing the inverse table should require very little total time compared to the memory accesses needed to carry out the proof of effort.

The limitation of this approach is that, since $g$ can be computed with no memory accesses, there is a time/space tradeoff for inverting $g$ in which no memory accesses are performed (in our context, space refers to cache size, since we are interested in what can be done without going to memory) [21.1426]. Those results imply that $g$ can be inverted at the cost of two forward computations of $g$, with no memory accesses.

This suggests basing computational challenges on functions that are (in some sense) hard in both directions.

\section{An Abstract Function and Lower Bound on Cache Misses}

In this section we describe an "abstract" pricing function and prove a tight lower bound on the number of memory accesses that must be made in order to produce a message acceptable to the receiver, in the model defined in Section 3 . The function is "abstract" in that it uses idealized hash functions, also known as random oracles. A concrete implementation is proposed in Section 6

Meaning of the Model and the Abstraction: Our computational model implicitly constrains the adversary by constraining the architecture available to the adversary. Our use of random oracles for the lower bound argument similarly constrains the adversary, as there are some things it cannot compute without accessing the oracles. We see two

\footnotetext{
${ }^{4}$ The root of the tree is labelled with $y$. A vertex at distance $d \geq 0$ from the root having label $z \in \operatorname{Range}\left(g_{\ell-d}\right)$ has one child labeled with each $z^{\prime} \in g^{-1}(z \oplus(\ell-d)) \in \operatorname{Range}\left(g_{\ell-d-1}\right)$.
} 
advantages in such modelling: (i) It provides rationale to the design of algorithms such as those of Section 6, this is somewhat similar to what Luby and Rackoff [22] did for the application of Feistel Permutations in the design of DES; (ii) If there is an attack on the simplified instantiation of the algorithm of Section 6 , then the model provides guidelines for modifications. Note that we assume that the arguments to the random oracle must be in cache in order to make the oracle call.

The inversion techniques of [21,14] do not apply to truly random functions, as these have large Kolmogorov complexity (no short representation). Accordingly, our function involves a large fixed forever table $T$ of truly random $w$-bit integers 5 . The table should be approximately twice as large as the largest existing caches, and will dominate the space needs of our memory-bound function.

We want to force the legitimate sender of a message to take a random walk "through $T$," that is, to make a series of random accesses to $T$, each subsequent location determined, in part, by the contents of the current location.

Such a walk is called a path. The algorithm forces the sender to explore many different paths until a path with certain desired characteristics is found. We call this a successful path. Once a successful path has been identified, information enabling the receiver to check that a successful path has been found is sent along with the message. Verification requires work proportional to the path length, determined by a parameter $\ell$. Each path exploration is called a trial. The expected number of trials to find a successful path is $2^{e}$, where $e$ (for "effort") is a parameter. The expected amount of work performed by the sender is proportional to $2^{e}$ times the path length.

\subsection{Description of the Abstract Algorithm}

The algorithm uses a modifiable array $A$, initialized for each trial, of size $|A| w>b$ bits (recall that $b$ is the number of bits in a memory block, or cache line)

Before we present the abstract algorithm, we introduce a few hash functions $H_{0}, H_{1}$, $\mathrm{H}_{2}, \mathrm{H}_{3}$, of varying domains and ranges, that we model as idealized random functions (random oracles). The function $H_{0}$ is only used during initialization of a path. It takes as input a message $m$, sender's name (or address) $S$, receiver's name (or address) $R$, and date $d$, together with a trial number $k$, and produces an array $A$. The function $H_{1}$ takes an array $A$ as input and produces an index $c$ into the table $T$. The function $H_{2}$ takes as input an array $A$ and an element of $T$ and produces a new array, which gets assigned to $A$. Finally, the function $H_{3}$ is applied to an array $A$ to produce a string of $4 \mathrm{w}$ bits.

A word on notation: For arrays $A$ and $T$, we denote by $|A|$ (respectively, $|T|$ ) the number of elements in the array. Since each element is a word of $w$ bits, the numbers of bits in these arrays are $|A| w$ and $|T| w$, respectively.

The path in a generic trial is given by:

\footnotetext{
5 "Fixed forever" means fixed until new machines have bigger caches, in which case the function must be updated.

${ }^{6}$ The intuition for requiring $|A| w>b$ is that, since $A$ cannot fit into a single memory block, it is more expensive to fetch $A$ into cache than it is to fetch an element of $T$ into cache.
} 
Initialization:

$A=H_{0}(m, R, S, d, k)$

Main Loop: Walk for $\ell$ steps ( $\ell$ is the path length):

$$
c \leftarrow H_{1}(A)
$$

$A \leftarrow H_{2}(A, T[c])$

Success occurs if:

after $\ell$ steps the last $e$ bits of $H_{3}(A)$ are all zero.

Path exploration is repeated for $k=1,2, \ldots$ until success occurs. The information for identifying the successful path is simply all five parameters and the final $H_{3}(A)$ obtained during the successful tria 7 .

Verification that the path is indeed successful is trivial: the verifier simply carries out the exploration of the one path and checks that success indeed occurs with the given parameters and that the reported hash value $H_{3}(A)$ is correct.

The connection to Algorithm MBound, described in Section 6 , will be clear: we need only specify the four hash functions. To keep computation costs low in MBound, we will not invoke full-strength cryptographic functions in place of the random oracles, nor will we even modify all entries of array $A$ at each step.

The size of $A$ also needs consideration. If $A$ is too small, say, a pointer into $T$, then the spammer can mount an attack in which many different paths (trials for either the same or different messages) can be explored at a low amortized cost, as we now informally describe. At any point, the spammer can have many different $A$ 's (that is, $A$ 's for different trials) in the cache. The spammer then fetches a memory block containing several elements of $T$, and advances along each path for which some element in the given memory block was needed. This allows exploitation of locality in $T$. Thus, intuitively, we should choose $|A|$ sufficiently large that it is infeasible to store many different $A$ 's in the cache.

\subsection{Lower Bound on Cache Misses}

We now prove a lower bound on the amortized number of block transfers that any adversary constrained as described in Section 3 must incur in order to find a successful path. Specifically, we show that the amortized complexity (measured in the number of memory fetches per message) of the abstract algorithm is asymptotically tight.

The computation on each message must follow a specific sequence of oracle calls in order to make progress. The adversary may make any oracle calls it likes; however, to make progress on a path it must make the specified calls. By watching an execution unfold, we can observe when paths begin, and when they make progress. Calls to the oracle that make progress (as determined by the history) are called progress calls.

Theorem 1. Consider an arbitrarily long but finite execution of the adversary's program - we don't know what the program is, only that the adversary is constrained to use an architecture as described in Section 3 Under the following additional conditions, the amortized complexity of generating a proof of effort that will be accepted by a verifier is $\Omega\left(2^{e} \ell\right)$ :

\footnotetext{
${ }^{7}$ The value of $H_{3}(A)$ is added to prevent the spammer from simply guessing $k$, which has probability $1 / 2^{e}$ of success.
} 
- $|T| \geq 2 s$ (recall that the cache contains $s$ words of $w$ bits each)

- $|A| w \geq b s^{1 / 5}$ (recall that $b$ is the block size, in bits).

$-\ell>8|A|$

- The total amount of work by the spammer (measured in oracle calls) per successful path is no more than $2^{o(w)} 2^{e} \ell$.

- $\ell$ is large enough so that the spammer cannot call the oracle $2^{\ell}$ times.

Remark 3. First note that $|A|$ is taken to be much larger than $b / w$. We already noted that if $|A|$ is very small than a serious attack is possible. However, even if $|A|$ is roughly $b / w$, it is possible to attack the algorithm by storing many copies of $T$ under various permutations. In this case the adversary can hope to concurrently be exploring about $\log s$ paths for which a single memory block contains the value in $T$ needed by all $\log s$ paths. Hence, if (for some reason) it is important that $|A| \leq O(b / w)$ we can only get a lower bound of the form $\Omega\left(2^{e} \ell / \log s\right)$.

Proof. (of Theorem $1 \mathrm{We}$ start with an easy lemma regarding the number of oracle calls needed to find a successful path.

Lemma 1. The amortized number of calls to $H_{1}$ and $H_{2}$ per proof of effort that will be accepted by a verifier is $\Omega\left(2^{e} \ell\right)$.

Lemma 2. Let $b_{1} \ldots b_{m}$ be independent unbiased random bits and let $k \leq m$. Suppose we have a system that, given a hint of length $B<k$ (which may be based on the value of $\left.b_{1} \ldots b_{m}\right)$, produces a subset $S$ of $k$ indices and a guess of the values of $\left\{b_{i} \mid i \in S\right\}$. Then the probability that all $k$ guesses are correct is at most $2^{B} / 2^{k}$, where the probability is over the random variables and the coin flips of the hint generation and the guessing system.

We now get to the main content of the lower bound and to the key lemma (Lemma 3): We break the execution into intervals in which, we argue, the adversary is, forced to learn a large number of elements of $T$. That is, there will be a large number of scattered elements of $T$ which the adversary will need in order to make progress during the interval, and very little information about these elements is in the cache at the start of the interval.

We first motivate our definition of an interval. We want to think of each $A$ as incompressible, since it is the output of a random function. However, if, say, this is the beginning of a path eploration, and $A=H_{0}(m, S, R, d, k)$, then it may require less space simply to list the arguments to $H_{0}$; since our model does not charge (much) for oracle calls, the adversary incurs no penalty for this. For this reason, we will focus on the values of $A$ only in the second half of a path. Recall that $A$ is modified at each step of the Main Loop; intuitively, since these modifications require many elements of $T$, these "mature" A's cannot be compressed. Our definition of an interval will allow us to focus on progress on paths with "mature" $A$ 's.

Let $n=s /|A|$; it is helpful to think of $n$ as the number of $A$ 's that can simultaneously fit into cache (assuming they are incompressible). A progress call is mature if it is the $j$ th progress call of the path, for $j>\ell / 2$ (recall that $\ell$ is the length of a path). An interval is defined by fixing an arbitrary starting point in an execution of the adversary's 
algorithm (which may involve the simultaneous exploration of many paths), and running the execution until $8 n$ mature progress calls (spread over any number of paths) have been made to oracle $H_{1}$.

Lemma 3. The average number of memory accesses made during an interval is $\Omega(n)$, where the average is taken over the choice of $T$, the responses of the random oracles, and the random choices made by the adversary.

It is an easy consequence of this lemma that the amortized number of memory accesses to find a successful path is $\Omega\left(2^{e} \ell\right)$. This is true since by Lemma 1 success requires an expected $\Omega\left(2^{e} \ell\right)$ mature progress calls to $H_{1}$, and the number of intervals is the total number of mature progress calls to $H_{1}$ during the execution, divided by $8 n$, which is $\Omega\left(2^{e} \ell / n\right)$. (Note that we have made no attempt to optimize the constants involved.)

Proof. (of Lemma 3) Intuitively, the spammer's problem is that of asymmetric communication complexity between memory and the cache. Only the cache has access to the functions $H_{1}$ and $H_{2}$ (the arguments must be brought into cache in order to carry out the function calls). The goal of the (spammer's) cache is to perform any $8 n$ mature progress calls. Since by definition the progress calls to $H_{1}$ are calls in which the arguments have not previously been given to $H_{1}$ in the current execution, we can assume the values of $H_{1}$ 's responses on these calls are uniform over $\{1, \ldots,|T|\}$ given all the information currently in the system (memory and cache contents and queries made so far). The cache must tell the memory which blocks are needed for the subsequent call to $\mathrm{H}_{2}$. Let $\beta$ be the average number of blocks sent by the main memory to the cache during an interval, and we assume for the sake of contradiction that $\beta=o(n)$ (the lemma asserts that $\beta=\Omega(n)$ ). We know that the cache sends the memory $\beta \log m$ bits to specify the block numbers (which is by assumption $o(n \log m)$ bits), and gets in return $\beta b$ bits altogether from the memory. The key to the lemma is, intuitively, that the relatively few possibilities in requesting blocks by the cache imply that many different elements of $T$ indicated by the indices returned by the $8 n$ mature calls to $H_{1}$ have to be stored in the same set of blocks. We will argue that this implies that a larger than $s$ part of $T$ can be reconstructed from the cache contents alone, which is a contradiction given the randomness of $T$.

We now proceed more formally. Lemma 3 will follow from a sequence of claims. The first is that there are many entries of $T$ for which many possible values are consistent with the cache contents at the beginning of the interval. That is, $T$ is largely unexplored from the cache's point of view. The proof is based on Sauer's Lemma (see [6])

Claim 1. There exist $\gamma, \delta \geq 1 / 2$ such that: given the cache contents at the beginning of the interval, it is expected that there exists a subset of the entries of $T$, called $T^{\prime}$, of size at least $\delta|T|$ such that for each entry $i$ in $T^{\prime}$ there is a set $S_{i}$ of $2^{\gamma w}$ possible values for $T[i]$ and all the $S_{i}$ 's are mutually consistent with the cache contents.

From now on we assume that we have cache content consistent with a large number of possibilities for $T^{\prime}$ as in the claim and use this cache configuration to show that it is possible to extract many entries of $T^{\prime}$.

Claim 2. If the number $\beta$ of memory accesses is o( $n)$, then the number of different paths on which a mature progress call is made during an interval is at most $3 n$. 
It therefore follows that in a typical interval there are at least $8 n-3 n=5 n$ pairs of consecutive mature progress calls to $H_{1}$ on a common path. Thus, for example, one path may experience $5 n+1$ mature progress calls, or each of $n$ paths may experience at least 6 mature progress calls, or something in between. Each such pair of calls to $H_{1}$ is separated by a call to $H_{2}$ which requires the contents of the location of $T$ specified by the first $H_{1}$ call in the pair. It is these interstitial calls to $H_{2}$ that are of interest to us: because their preceding calls to $H_{1}$ first occur during the interval, and $H_{1}$ is random, it cannot be known at the start of the interval which elements of $T$ will be needed as arguments to these calls to $\mathrm{H}_{2}$. Intuitively, the adversary must go to main memory to find an expected $(|T|-s) /|T|>1 / 2$ of them.

Consider the set of $8 n$-tuples over $\{1, \ldots,|T|\}$ as the set of possible answers $H_{1}$ returns on the mature progress calls in the interval; there are $|T|^{8 n}$ such tuples. Fix all other random choices: the value of $T$, the previous calls to $H_{1}$ and $H_{2}$ and the random tape of the spammer). The spammer's behavior in an interval is now determined solely by this $8 n$-tuple. If the spammer can defeat our algorithm, then, for some fixed $\epsilon>0$, the spammer completes the interval retrieving at most $2 \beta$ blocks with at least $\epsilon$ probability, over the choice of $8 n$-tuple. Call these tuples the good ones. By Markov's inequality, for at least half of these good $8 n$-tuples the spammer retrieves at least $\beta$ blocks. We first claim that in most of those tuples the spammer goes frequently into $H_{2}$ with values $T[i]$ where $i \in T^{\prime}$.

Claim 3. Let $T^{\prime}$ be any subset of the entries of $T$ of size at least $\delta n$. Consider the set of good 8 -tuples over $\{1, \ldots,|T|\}$ as the set of possible answers $H_{1}$. Then except for at most an exponential in $n$ fraction of them the spammer must use an entry in $T^{\prime}$ for a call to at $\mathrm{H}_{2}$ least $n$ times during an interval.

Claim 4. Suppose that we have subset $X$ of size $x$ of entries in $T$. Then the probability over $H_{1}$ that a $8 n$-tuple contains more than $n / 2$ entries in $X$ is at most $\left(2^{8} x /|T|\right)^{n / 2}$.

Claim 5. Suppose that we have a collection of good 8 -tuples and we want to cover at least $x$ values in $T^{\prime}$ using only a few members of the collection, say $2 x / n$ (assume that the collection is at least that large). If this is impossible then there is a set $X \subset T^{\prime}$ of size $x$ such that every member of the collection has at least $n / 2$ entries in $X$.

The idea for deriving the contradiction to the fact that only $\beta=o(n)$ blocks are brought from memory to cache is that there should be many good $8 n$-tuples that share the same set of blocks (that is, by retrieving one set of blocks all elements appearing in many good $8 n$-tuples can be reconstructed in the cache). In fact, since the memory size is $m$, a $1 /\left(\begin{array}{c}m \\ 2 \beta\end{array}\right)$ fraction of them share the same set of blocks (the factor of 2 comes from the definition of a good $8 n$-tuple). Consider such a collection and suppose that there are $2 x / n$ tuples in this collection whose union covers $x$ entries in $T^{\prime}$. Then the "memory" can use these $2 x / n$ tuples to transfer the value of $x$ entries in $T^{\prime}$ by sending the $2 \beta b$ bits describing the content of the common blocks and in addition for each tuple in the cover: (1) Specifying the $8 n$-tuple: this takes $8 n \log |T|$ bits; (2) Specifying which calls to $H_{2}$ in the execution have the correct parameters (there may be some "bogus" calls to 
$\mathrm{H}_{2}$ in which the wrong values for elements of $T$ are used as parameters). If the interval contains $z$ calls to $H_{2}$ then this takes $\log \left(\begin{array}{c}z \\ n / 2\end{array}\right)$ bits which is $O(n \log z)$.

So altogether it suffices for $2 \beta b+16 x \log |T|+2 x \log z$ bits to be sent from the memory to the cache. In return, the cache learns $\gamma w$ bits for each of $x$ entries in $T^{\prime}$, or $x \gamma w$ bits altogether. To derive the contradiction, since $w$ was taken to be much larger than $\log |T|$ and $2^{w / 2}$ much larger than the amortized number of oracle calls per interval, $\log z$ is much smaller than $w$ and we only have to worry about the $2 \beta b$ term.

Assume that $\beta \leq 1 / 20 n=s / 20|A|$ and, for simplicity that $m$, the memory size is $|T|^{2}$ (recall that in our model $m$ is polynomial in $s$, and in our theorem $|T|=\Theta(s)$ ). Set $x=4 \beta b / w$. Of all good tuples, pick the largest collection agreeing with a set of $\beta$ blocks, i.e., consisting of at least a $1 /\left(\begin{array}{c}T^{2} \\ \beta\end{array}\right)$ fraction of the good tuples. We now claim that this collection has $2 x / n 8 n$-tuples whose union is of size at least $x$ (this will be sufficient for a contradiction).

Suppose that this is not the case and the $2 x / n$ tuples covering $x$ do not exist. Then as we have seen above in Claim[5 there is a set $X$ of size $x$ where each tuple in the collection has at least $n / 2$ entries in $X$. But we know from Claim 4 that the fraction (among all tuples) of such a collection can be at most $\left(2^{8} x /|T|\right)^{n / 2}$. Taking into account $\epsilon$ (the faction of all tuples that are good) we must compare $\left(\left(2^{8} x /|T|\right)^{n / 2}\right)(1 / \epsilon)$ to $1 /\left(\begin{array}{c}T^{2} \\ 2 \beta\end{array}\right)$ and if the latter is larger we know that the collection is too large to be compressed into $X$. For simplicity take $\epsilon=1$. Indeed

$$
\frac{\left(2^{8} x /|T|\right)^{n / 2}}{\left(\begin{array}{c}
T^{2} \\
2 \beta
\end{array}\right)}=\frac{\left(2^{8} x\right)^{n / 2}}{T^{n / 2-4 \beta}}
$$

taking $\log$ s we get that we need to compare $\log 2^{8} x$ and

$$
(\log T) \frac{n-4 \beta}{n}=(\log T) \frac{s /|A|-4 s / 20|A|}{s /|A|}=(\log T) \frac{4}{5} .
$$

But since $x=4 \beta b / w=4 s b /(20|A| w)$ and $|A| \geq s^{1 / 5} b / w$ we get that $x \leq 1 / 5 s^{4 / 5}$ and indeed $8+\log x$ is smaller than $4 / 5 \log |T|$.

This concludes the proofs of Lemma 3 and Theorem 1

\section{A Concrete Proposal}

In this section we describe a concrete implementation of the abstract algorithm of Section 5. which we call Algorithm MBound. As in the abstract algorithm, our function involves a large fixed forever array $T$, now of $2^{22}$ truly random 32-bit integer\$. In terms of the parameters of Section [5] we have $|T|=2^{22}$ and $w=32$. This array requires 16 $\mathrm{MB}$ and dominates the space needs of our memory-bound function, which requires less than $18 \mathrm{MB}$ total space 9 The algorithm requires in addition a fixed-forever truly random array $A_{0}$ containing 25632 -bit words. $A_{0}$ is used in the definition of $H_{0}$. Note that $A_{0}$ is incompressible.

\footnotetext{
8 "Fixed forever" means fixed until new machines have bigger caches, in which case the function must be updated.

${ }^{9}$ To send mail, a machine must be able to handle a program of this size.
} 


\subsection{Description of MBound}

Our proposal was inspired by the (alleged) RC4 pseudo-random generator (see, e.g., the descriptions of RC4 in [16/23,24]).

Description of $H_{0}$. Recall that we have a fixed-forever array $A_{0}$ of 256 truly random 32-bit words. At the start of the $k$ th trial, we compute $A=H_{0}(m, S, R, d, k)$ by first computing (using strong cryptography) a 256-word mask and then XORing $A_{0}$ together with the mask. Here is one way to define $H_{0}$ :

1. Let $\alpha_{k}=h(m, S, R, d, k)\left(\left|\alpha_{k}\right|=128\right)$, for a cryptographically strong hash function $h$ such as, say, SHA-1.

2. Let $\eta\left(\alpha_{k}\right)$ be the $2^{13}$-bit string obtained by concatenating the $2^{7}$-bit $\alpha_{k}$ with itself $2^{6}$ times 10 . Treating the array $A$ as a $2^{13}$-bit string (by concatenating its entries in row-major order), we let $A=A_{0} \oplus \eta\left(\alpha_{k}\right)$. Note that, unlike in the case of RC4, our array $A$ is not a permutation of elements $\{1,2, \ldots, 256\}$, and its entries are 32 bits, rather than 8 bits.

We initialize $c$, the current location in $T$, to be the last 22 bits of $A$ (when $A$ is viewed as a bit string). In the sequel, whenever we say $A[i]$ we mean $A\left[i \bmod 2^{8}\right]$; similarly, by $T[c]$ we mean $T\left[c \bmod 2^{22}\right]$.

The path in a generic trial is given by:

Initialize Indices:

$$
i=0 ; j=0
$$

Walk for $\ell$ steps ( $\ell$ is the path length):

$i=i+1$

$j=j+A[i]$

$A[i]=A[i]+T[c]$

$A[i]=$ RightCyclicShift $(A[i], 11)$ (shift forces all 32 bits into play)

$$
\begin{aligned}
& \operatorname{Swap}(A[i], A[j]) \\
& c=T[c] \oplus A[A[i]+A[j]] \\
& \text { Success occurs if the last } e \text { bits of } \mathrm{h}(\mathrm{A}) \text { are all } 0 .
\end{aligned}
$$

In the last line, the hash function $h$ can again be SHA-1. It is applied to $A$, treated as a bit string.

The principal difference with the RC4 pseudo-random generator is in the use of $T$ : bits from $T$ are fed into MBound's pseudo-random generation procedure, both in the modification of $A$ and in the updating of $c$.

In terms of the abstract function, we can tease our proposal apart to obtain, roughly:

Description of $H_{1}$ (updates $c$, leaves $A$ unchanged). The function $H_{1}$ is essentially

$$
\begin{aligned}
& i=i+1 \\
& j=j+A[i]
\end{aligned}
$$

\footnotetext{
${ }^{10}$ The reason we concatenate the string in order to generate $\eta\left(\alpha_{k}\right)$, rather than generate a cryptographically strong string of length $2^{13}$ is to save CPU cycles - this is an operation that is done many times and if each bit of $\eta\left(\alpha_{k}\right)$ is strong it could make the scheme CPU bound.
} 


$$
\begin{aligned}
& v=A[i]+T[c] \quad(v \text { is a temporary variable) } \\
& v=\operatorname{RightCyclicShift}(v, 11) \\
& c=T[c] \oplus A[A[j]+v]
\end{aligned}
$$

Description of $\mathrm{H}_{2}$ (updates $A$ ).

$$
\begin{aligned}
& A[i]=A[i]+T[c] \\
& A[i]=\text { RightCyclicShift }(A[i], 11) \\
& \operatorname{Swap}(A[i], A[j])
\end{aligned}
$$

Description of $H_{3}$. The hash function $H_{3}(A)$ is simply some cryptographically strong hash function with 128 bits of output, such as SHA-1.

This all but completes the description of Algorithm MBound and its connection to our abstract function; it remains to choose the parameters.

\subsection{Parameters for MBound}

We can define the computational puzzle solved by the sender as follows.

Input. A message $m$, a sender's alias $S$, a receiver's alias $R$, a time $t$, the table $T$ and the auxiliary table $A_{0}$.

Output. $m, S, R, d, i$ and $\alpha$ such that $1 \leq i \leq 2 e$ and the $i$ th path (that is, the path with trial number $k=i$ ), is successful and $\alpha$ is the result of hashing the final value of $A$ in the successful path.

If $i>2^{2 e}$, the receiver rejects the message (with overwhelming probability one of the first $2^{2 e}$ trials should be successful).

To be specific in the following analysis, we make several assumptions. These assumptions are reasonable for current technology, and our analysis is sufficiently robust to tolerate substantial changes in many of these parameters. Let $P$ be the desired expected time for computing the proof of effort and let $\tau$ be the memory latency. We assume that $P$ is 10 seconds and $\tau$ is .2 microseconds. We also assume that the maximum size of the fast cache is $8 \mathrm{MB}$ and that cache lines (memory blocks) are 64 bytes wide (so blocks contain $b=512$ bits).

The output conditions ensure that for a random starting point, the probability of a successful output is $1 / 2^{e}$. The expected number of walks to be checked is $2^{e}$. Therefore the expected value of $P$ is

$$
E[P]=2^{e} \cdot \ell \cdot \tau .
$$

The cost of verification by the receiver is essentially $\ell$ cache misses, by following the right path. (In Section 8 we discuss how to reduce or eliminate these cache misses.)

We have not yet set the values for $e$ and $\ell$. Choosing one of these parameters forces the value of the other one. Consider the choice of $e$ : one possibility might be to make $e$ very large, and the paths short, say, even of length 1 . This would make verification extremely cheap. However, while the good sender will explore the paths sequentially, a cheating sender may try several paths in parallel, hoping to exploit locality by batching several accesses to $T$, one from each of these parallel explorations. In addition, $A$ changes slowly, and to get to the point in which many "mature" values of $A$ cannot be compressed requires that many entries of $A$ have been modified. For our concrete proposal, therefore, we let $\ell=2048$. Then $2^{e}=P / \ell \tau=10 /\left(2048 * 2 * 10^{-7}\right) \approx 24,414$. 
Table 1. Computational Platforms, sorted by CPU speed.

\begin{tabular}{|l|l|crcc|}
\hline name & lass & model & processor CPU clock & OS \\
\hline P4-3060 & workstation & DELL XW8000 & Intel Pentium 4 & $3.06 \mathrm{Ghz}$ & Linux \\
P4-2000 & desktop & Compaq Evo W6000 & Intel Pentium 4 & $2.0 \mathrm{Ghz}$ & Windows XP \\
P3-1200 & laptop & DELL Latitude C610 & Intel Pentium 3M & $1.2 \mathrm{Ghz}$ & Windows XP \\
P3-1000 & desktop & Compaq DeskPro EN & Intel Pentium 3 & $1.0 \mathrm{Mhz}$ & Windows XP \\
Mac-1000 & desktop & Power Mac G4 & PowerPC G4 & $1000 \mathrm{Mhz}$ & OSX \\
P3-933 & desktop & DELL Dimension 4100 & Intel Pentium 3 & $933 \mathrm{Mhz}$ & Linux \\
SUN-900 & server & SUN Ultra 60 & UlraSPARC III+ & $900 \mathrm{Mhz}$ & Solaris \\
SUN-450 & server & SUN Ultra 60 & UlraSPARC II & $450 \mathrm{Mhz}$ & Solaris \\
P2-266 & laptop & Compaq Armada 7800 & Intel Pentium 2 & $266 \mathrm{Mhz}$ & Windows 98 \\
S-233 & settop & GCT-AllWell STB3036N Nat. Semi. Geode GX1 & $233 \mathrm{Mhz}$ & Linux \\
\hline
\end{tabular}

\section{Experimental Results}

In this section we describe several experiments aimed at establishing practicality of our approach and verifying it experimentally. First we compare our memory-bound function performance to that of the CPU-intensive HashCash function [18] on a variety of computer architectures. We confirm that the memory-bound function performance is significantly more platform-independent. We also measure the solution-to-verification time ratio of our function. Then we run simulations showing how the number of cache misses during the execution of our memory-bound function depends on the cache size and the cache replacement strategy. We observe that even if an adversary knows future accesses, this does not help much unless the cache size is close to the size of $T$. Finally, we study how the running time depends on the size of the big array $T$.

Table 2. Memory hierarchy.

\begin{tabular}{|c|c|c|c|}
\hline machine & L2 cache & L2 line & memory \\
\hline P4-3060 & $256 \mathrm{~KB}$ & 128 bytes & $4 \mathrm{~GB}$ \\
\hline P4-2000 & $256 \mathrm{~KB}$ & 128 byt & $512 \mathrm{MB}$ \\
\hline P3-1200 & $256 \mathrm{~KB}$ & 64 byt & MB \\
\hline P3-1000 & $256 \mathrm{~KB}$ & 64 bytes & $512 \mathrm{MB}$ \\
\hline P3-933 & $256 \mathrm{~KB}$ & 64 bytes & $512 \mathrm{MB}$ \\
\hline Mac-1000 & $256 \mathrm{~KB}$ & 64 bytes & $512 \mathrm{MB}$ \\
\hline SUN-900 & $8 \mathrm{MB}$ & 64 bytes & $8 \mathrm{~Gb}$ \\
\hline SUN-450 & $8 \mathrm{MB}$ & 64 bytes & $1 \mathrm{~Gb}$ \\
\hline P2-266 & $512 \mathrm{~KB}$ & 32 bytes & $96 \mathrm{MB}$ \\
\hline S-233 & $16 \mathrm{~KB}$ & 16 bytes & $128 \mathrm{MB}$ \\
\hline
\end{tabular}

\subsection{Different Architectures}

We conducted tests on a variety of platforms, summarized in Table 1 These platforms vary from the popular Pentium 3 and Pentium 4 systems and a Macintosh G4 to SUN servers with large caches. We even tested our codes on a settop box, which is an example 
of a low-power device. The P2-266 laptop is an example of a "legacy" machine and is representative of a low-end machine among those widely used for e-mail today (that is, in 2003). Table 2 gives sizes of the relevant components of the memory hierarchy, including L2 cache size, L2 cache line size, and memory size. With one exception, all machines have two levels of cache and memory. The exception is the Macintosh, which has a 2 MB off-chip L3 cache in addition to the $256 \mathrm{~KB}$ on-chip L2 cache.

Table 3. Program timings. Times are averages over 20 runs, measured in units of the smallest average. For HashCash, the smallest average is $4.44 \mathrm{sec}$; for MBound, it is $9.15 \mathrm{sec} \%$.

\begin{tabular}{|c|c|c|}
\hline $\begin{array}{c}\text { machine } \\
\text { name }\end{array}$ & $\begin{array}{r}\text { HashCash } \\
\text { time }\end{array}$ & $\begin{array}{l}\text { MBound } \\
\text { time sol./ver. }\end{array}$ \\
\hline P4-3060 & 1.00 & $1.012 .32 \mathrm{E} 4$ \\
\hline P4-2000 & 1.91 & $1.331 .65 \mathrm{E} 4$ \\
\hline P3-1200 & 2.21 & $1.002 .55 \mathrm{E} 4$ \\
\hline P3-1000 & 2.67 & $1.062 .48 \mathrm{E} 4$ \\
\hline Mac-1000 & 1.86 & $1.962 .61 \mathrm{E} 4$ \\
\hline P3-933 & 2.15 & $1.062 .51 \mathrm{E} 4$ \\
\hline SUN-900 & 1.82 & $2.242 .50 \mathrm{E} 4$ \\
\hline SUN-450 & 5.33 & $2.942 .02 \mathrm{E} 4$ \\
\hline P2-266 & 10.17 & $2.671 .84 \mathrm{E} 4$ \\
\hline $\mathrm{S}-233$ & 43.20 & $4.621 .50 \mathrm{E} 4$ \\
\hline
\end{tabular}

\subsection{Memory- vs. CPU-Bound}

The motivation behind memory-bound functions is that their performance is less dependent on processor speed than is the case for CPU-bound functions. Our first set of experiments compares an implementation of our memory-bound function, MBound, to our implementation of HashCash [18]. HachCash repeatedly appends a trial number to the message and hashes the resulting string, until the output ends in a certain number zero bits (22 in our experiments). For MBound, with its slower iteration time, we set the required number of zero bits to 15 .

Table 3 gives running times for HashCash and MBound, normalized by the fastest machine time. Note that HashCash times are closely correlated with processor speed. Running times for MBound show less variation. The difference between the P2-266 laptop and the fastest machine used in our tests for HashCash is a factor of 10.17, while the difference for MBound is only a factor of 2.67. The HashCash vs. MBound gap is even larger for the S-233 settop box 11

Modern Pentium-based machines perform well in memory-bound computations. The Macintosh does not do so well; we believe that this is due to its poor handling of the translation lookahead buffer (TLB) misses. SUN servers do poorly in spite of their large

\footnotetext{
${ }^{11}$ Note that $\mathrm{S}-233$ is a special-purpose device and code produced by the $\mathrm{C}$ compiler may be poorly optimized of the processor. This may be one of the reasons why this machine was so slow in our tests.
} 
caches. This is due to their poor handling of TLB misses and the penalty for their ability of handle large memories.

\section{Freeing the Receiver from Accessing $T$}

Since the spam-protected receiver will sometimes act also as an e-mail sender, he will have access to the array $T$. However, we would like receiving mail not to have to involve accessing $T$ at all. For example, one might wish to be able to receive mail on a cell phone. In this section we explore the possibility that the sender adds some information to its message that will permit the receiver to efficiently verify the proof of effort with no accesses to $T$. Of course, the conceptually simplest method for freeing $R$ from accessing $T$ is for the creator of $T$ to sign all the elements of $T$ (more precisely, the signature is on the pair $(c, T[c])$, to disallow permuting the table). However, this requires too much storage at the sender, even using the signature scheme yielding the shortest signatures [8].

Compressed RSA Signatures. Here we use properties of the RSA scheme previously exploited in the literature [13,12]. Let $(N, e)$ be the public key of an RSA signature scheme chosen by the creator of $T^{12}$. Let $F$ be a function mapping pairs $(c, T[c])$ into $Z_{N}^{*}$, that is, a mapping from $32+22=54$-bit strings into $Z_{N}^{*}$. In our analysis we will model $F$ as a random oracle. For all $1 \leq c \leq|T|$ let $v_{c}=F(c, T[c])$ and let $w_{c}=v_{c}^{1 / e} \bmod N$. Thus, $v_{c}$ is a hash of the pair $(c, T[c])$ and $w_{c}$ is a signature on the string $v_{c}$.

The sender's protocol contains, in addition to $T$, the public modulus $N$, the description of $F$, and the $w_{c}$ 's. The receiver's protocol uses only the description of $F$ and the public key $(N, e)$, together with a description of the sender's path exploration algorithm (minus the array $T$ itself).

Let the sender's successful path be the sequence $c_{1}, c_{2}, \ldots c_{\ell}$ of locations in $T$. The proof of effort contains two parts:

1. $T\left[c_{1}\right], T\left[c_{2}\right], \ldots, T\left[c_{\ell}\right]$, (a total of about $\left.4 \mathrm{~KB}\right)$, and

2. $w=\prod_{i=1}^{\ell} w_{c_{i}} \bmod N($ about $1 \mathrm{~KB})$.

Note that there is no need to include the indices $c_{1}, \ldots, c_{\ell}$ in the first part, as these are implicit from the algorithm. Similarly, there is no need to send the $v_{c}$ 's, since these are implicit from $F$ and the $\left(c_{i}, T\left[c_{i}\right]\right)$ pairs. Let $t_{1}, \ldots, t_{\ell}$ be the first part of the proof, and $w$ the second part (each $t_{i}$ is supposed to be $T\left[c_{i}\right]$, but the verifier cannot yet be certain this is the case). The proof is checked as follows.

1. Compute $v_{c_{1}}^{\prime}, v_{c_{2}}^{\prime}, \ldots v_{c_{\ell}}^{\prime}$, where $v_{c_{i}}^{\prime}=F\left(c_{i}, t_{i}\right)$.

2. Check whether $w^{e}=\left(\prod_{i=1}^{\ell} v_{c_{i}}\right) \bmod N$.

The security of the scheme rests on the fact that it is possible to translate a forged signature on

$$
\left(c_{1}, T\left[c_{1}\right]\right), \ldots\left(c_{\ell}, T\left[c_{\ell}\right]\right)
$$

$\overline{12}$ The signing key $d$ is a valuable secret! 
into an inversion of the RSA function on a specific instance. This is summarized as follows:

Theorem 2. If $F$ is a random oracle, then any adversary attempting to produce a set of claimed values

$$
T\left[c_{1}\right], T\left[c_{2}\right], \ldots T\left[c_{\ell}\right]
$$

that is false yet acceptable to the receiver can be translated into an adversary for breaking $R S A$ with the same run time and probability of success (to preserve probability of success we need that e be a prime larger than $\ell$ ).

Although transmission costs are low, the drawback of the compressed RSA scheme is again the additional storage requirements for the sender: each $w_{c}$ is at least 1,000 bits (note, however, that these extra values are not needed until after a successful path has been found). This extra storage requirement might discourage a user from embracing the scheme. We address this next.

Storage-Optimized Compressed RSA. We optimize storage with the following storage / communication / computation tradeoff: Think of $T$ as an $a \times b$ matrix where $a \cdot b=|T|$; the amount of extra communication will be $a$ elements of $T$. The amount of extra storage required by the sender will be $b$ signatures.

At a high level, given a path using values $T\left[c_{1}\right], T\left[c_{2}\right], \ldots, T\left[c_{\ell}\right]$, values in the same row of $T$ will be verified together as in the compressed RSA scheme. The communication costs will therefore be at most $a$ elements, one per row of $T$. However, as we will see below, there is no need to store the $w_{c}$ 's explicitly. Instead, we can get away with storing a relatively small number of signatures (one per column), from which it will be possible to efficiently reconstruct the $w_{c}$ values as needed.

Instead of a single exponent $e$, both sending and receiving programs will contain a (common) list of primes $e_{1}, e_{2}, \ldots e_{a}$. For $1 \leq i \leq a, e_{i}$ is used for verifying elements of row $i$ of the table. Although we don't need to store the $w_{c}$ values explicitly, for elements $v_{c}$ appearing in row $i$ we define $w_{c}=v_{c}^{1 / e_{i}} \bmod N$.

The compressed RSA scheme is applied to the entries in each row independently. It only remains to describe how the needed $w_{c}$ values are constructed on the fly.

The $b$ "signatures", one per column, used in the sending program are computed by the creator as follows. For each column $1 \leq j \leq b$, the value for column $j$ is $u_{j}=$ $\prod_{i=1}^{a} w_{c_{j_{i}}} \bmod N$. Here, $c_{j_{i}}$ is the index of the element $T[i, j]$, when $T$ is viewed as a matrix rather than as an array (that is, assuming row-major order, $\left.c_{j_{i}}=(i-1) a+j-1\right)$. Thus, $v_{c_{j_{i}}}=T[(i-1) a+j-1]$ and $w_{c_{j_{i}}}=\left(v_{c_{j_{i}}}\right)^{1 / e_{i}}$. As in Batch RSA [13], one can efficiently extract any $w_{c_{j_{i}}}$ from $u_{j}$ using a few multiplications and exponentiations.

Set $a=16$. The number of data bits in a column is $2^{4} \cdot 2^{5}=2^{9}$. The number of "signature bits" is $2^{10}$ per column. Thus storage requirement just more than doubles, rather than increasing by a factor of 5-10, at the cost of sending 16 elements of $Z_{N}^{*}$ (i.e., $2 \mathrm{~KB})$. 


\section{Concluding Remarks}

We have continued the discussion, initiated in [3], of using memory-bound rather than CPU-bound pricing functions for computational spam fighting. We considered and analyzed several potential approaches. Using insights gained in the analyses, we proposed a different approach based on truly random, incompressible, functions, and obtained both a rigorous analysis and experimental results supporting our approach.

From a theoretical perspective, however, the work is not complete. First, we have the usual open question that arises whenever random oracles are employed: can a proof of security (in our case, a lower bound on the average number of cache misses in a path) be obtained without recourse to random oracles? Second, much more unusually, can we prove security without cryptographic assumptions? Note that we did not make cryptographic assumptions in our analysis.

One of the more interesting challenges suggested by this work is to apply results from complexity theory in order to be able to make rigorous statements about proposed schemes. One of the more promising directions in recent years is the work on lower bounds for branching program and the RAM model by Ajtai s [45] and Beame et al [7]. It is not clear how to directly apply such results.

At first blush egalitarianism seems like a wonderful property in a pricing function. However, on reflection it may not be so desirable. Since the approach is an economic one it may be counterproductive to design functions that can be computed just as quickly on extremely cheap processors as on supercomputers - after all, we are trying to force the spammers to expend resources, and it is the volume of mail sent by the spammers that should make their lives intolerable while the total computational effort expended by ordinary senders remains benign. So perhaps less egalitarian is better, and users with weak or slow machines, including PDAs and cell phones, could subscribe to a service that does the necessary computation on their behalf. In any case, small-memory machines cannot be supported, since the large caches are so very large, so in any real implementation of computational spam fighting some kind of computation service must be made available.

\section{References}

1. M. Abadi and M. Burrows, (multiple) private communication(s)

2. M. Abadi, private communication.

3. M. Abadi, M. Burrows, M. Manasse and T. Wobber, Moderately Hard, Memory-Bound Functions, Proceedings of the 10th Annual Network and Distributed System Security Symposium, February, 2003.

4. M. Ajtai, Determinism versus Non-Determinism for Linear Time RAMs, STOC 1999, pp. 632-641.

5. M. Ajtai, A Non-linear Time Lower Bound for Boolean Branching Programs, FOCS 1999: 60-70

6. N. Alon, and J. Spencer, The Probabilistic Method, Wiley \& Sons, New-York, 1992

7. P. Beame, M. E. Saks, X. Sun, E. Vee, Super-linear time-space tradeoff lower bounds for randomized computation, FOCS 2000, pp. 169-179.

8. D. Boneh, B. Lynn and H. Shacham, Short signatures from the Weil pairing, ASIACRYPT 2001, pp. 514-532. 
9. www.camram.org/mhonarc/spam/msg00166.html.

10. W. Diffie and M.E. Hellman, Exhaustive cryptanalysis of the NBS Data Encryption Standard, Computer 10 (1977), 74-84.

11. C. Dwork and M. Naor, Pricing via Processing, Or, Combatting Junk Mail, Advances in Cryptology - CRYPTO’92, Lecture Notes in Computer Science No. 740, Springer, 1993, pp. 139-147.

12. C. Dwork and M. Naor, An Efficient Existentially Unforgeable Signature Scheme and Its Applications, Journal of Cryptology 11(3): 187-208 (1998)

13. A. Fiat, Batch RSA, . Journal of Cryptology 10(2): 75-88 (1997).

14. A. Fiat and M. Naor, Rigorous Time/Space Tradeoffs for Inverting Functions, STOC'91, pp. 534-541

15. A. Fiat and A. Shamir, How to Prove Yourself, Advances in Cryptoglogy - Proceedings of CRYPTO'84, pp. 641-654.

16. Fluhrer, I. Mantin and Shamir, Attacks on RC4 and WEP,. Cryptobytes 2002.

17. A. J. Menezes, P. C. van Oorschot and S. A. Vanstone, Handbook of Applied Cryptography, CRC Press, 1996. Also available: http://www.cacr.math.uwaterloo.ca/hac/

18. A. Back, Hashcash - A Denial of Servic Counter-Measure, available at http://www . cypherspace.org/hashcash/hashcash.pdf.

19. M. Bellare, J. A. Garay and T. Rabin, Fast Batch Verification for Modular Exponentiation and Digital Signatures, EUROCRYPT 1998, pp. 236-250.

20. M. Bellare, J. A. Garay and T. Rabin, Batch Verification with Applications to Cryptography and Checking, LATIN 1998, pp. 170-191.

21. M. Hellman, A Cryptanalytic Time Memory Trade-Off, IEEE Trans. Infor. Theory 26, pp. 401-406, 1980

22. M. Luby and C. Rackoff, How to Construct Pseudorandom Permutations and Pseudorandom Functions, SIAM J. Computing 17(2), pp. 373-386, 1988

23. I. Mantin, Analysis of the Stream Cipher RC4, Master's Thesis, Weizmann Institute of Science,

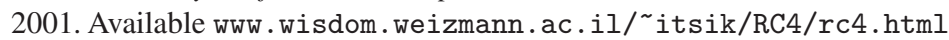

24. I. Mironov, (Not So) Random Shuffles of RC4, Proc. of CRYPTO'02, 2002

25. M. Naor and M. Yung, Universal One-Way Hash Functions and their Cryptographic Applications, STOC 1989, pp. 33-43

26. P. Oechslin, Making a faster Cryptanalytic Time-Memory Trade-Off, these proceedings.

27. P. C. van Oorschot and M. J. Wiener, Parallel Collision Search with Cryptanalytic Applications, Journal of Cryptology, vol. 12, no. 1, 1999, pp. 1-28.

28. R. Schroeppel and A. Shamir, A $T=O\left(2^{(n / 2)}\right), S=O\left(2^{(n / 4)}\right)$ Algorithm for Certain NP-Complete Problems, SIAM J. Comput. 10(3): 456-464 (1981). 1979. 